\title{
Các nhân tố ảnh hưởng đến việc áp dụng hệ thống thông tin bất động sản tại tỉnh Tiền Giang
}

\section{Factors affecting the application of real estate information system in Tien Giang province}

\author{
Nguyễn Minh Nhã ${ }^{{ }^{*}}$ \\ ${ }^{1}$ Trường Đại học Tiền Giang, Việt Nam \\ *Tác giả liên hệ, Email: nmn1312@gmail.com
}

THÔNG TIN

TÓM TÁT

DOI: $10.46223 / \mathrm{HCMCOUJS}$.
proc.vi.16.1.1852.2021

Ngày nhận: 7/3/2021

Ngày nhận lại: 30/3/2021

Duyệt đăng: 5/4/2021

Tù khóa:

hệ thống thông tin bất động sản, tỉnh Tiền Giang, nền tảng kỹ thuật, môi trường kinh doanh, dịch vụ hỗ trợ

Keywords:

real estate information system, Tien Giang province, technical platform, business environment, support services
Nghiên cứu này xác định sự ảnh hưởng của các nhân tố đến việc áp dụng hệ thống thông tin bất động sản tại tỉnh Tiền Giang. Đồng thời, tác giả cũng kiểm định việc áp dụng hệ thống thông tin bất động sản có thật sự mang lại lợi ích hay không? Dữ liệu nghiên cứu được thu thập từ 200 quan sát thực tế tại tỉnh Tiền Giang. Tác giả sử dụng mô hình phương trình cấu trúc (SEM) để phân tích dữ liệu thông qua việc đánh giá mô hình đo lường và mô hình cấu trúc. Kết quả nghiên cứu chỉ ra có 3 nhân tố là môi trường kinh doanh, nền tảng kỹ thuật và dịch vụ hỗ trợ có quan hệ thuận chiều đến việc áp dụng hệ thống thông tin bất động sản. Hơn nữa, kết quả nghiên cứu cho thấy việc áp dụng hệ thống thông tin bất động sản có quan hệ thuận chiều với lợi ích áp dụng.

ABSTRACT
This study determines the influence of these factors on the
application of real estate information system in Tien Giang
province. At the same time, we also test that the application of
the real estate information system is really beneficial or not?
Research data were collected from 200 actual observations in
Tien Giang province. We used structural equation modeling
(SEM) to analyze data through the evaluation of the measurement
model and structural model. Results research shows there are 3
factors as business environment, technical platform and support
services have positively related to the application of real estate
information systems. Moreover, the research results show that the
application of real estate information system has a positive
relationship with application benefits.

\section{Giới thiệu}

Ngày nay, bất động sản là loại tài sản luôn thu hút sự quan tâm của nhà đầu tư và người tiêu dùng. Việc thiếu thông tin hoặc thông tin không minh bạch sẽ ảnh hưởng đến các bên liên quan và có thể gây ra khủng hoảng bất động sản. Theo Konieczna (2017), bất động sản và thông tin về nó đã trở thành một yếu tố quan trọng cho sự phát triển kinh tế và hoạt động của một quốc gia. Do đó, mỗi quốc gia cần một hệ thống cung cấp thông tin minh bạch và bền vững về thị trường 
bất động sản.

Trên thế giới đã có nhiều nghiên cứu liên quan đến hệ thống thông tin về thị trường bất động sản. Fransson và Nelson (2000) đã xác định các yêu cầu về dữ liệu, hệ thống, người dùng và môi trường doanh nghiệp khi xây dựng và quản lý hệ thống thông tin về bất động sản. Honda, Sasaki, Hayashi, và Yasushi (2018) cho rằng việc đánh giá một hệ thống thông tin bất động sản phụ thuộc vào 3 nhân tố là môi trường vật chất, môi trường xã hội, giao thông và giao tiếp. Theo Konieczna (2017), việc áp dụng hệ thống thông tin bất động sản bị ảnh hưởng bởi 4 nhân tố là pháp lý, môi trường, công nghệ và kinh tế - xã hội. Thêm vào đó, nghiên cứu của Kirkwood (2004) cho thấy việc chấp nhận và áp dụng các đổi mới công nghệ thông tin cho thị trường bất động sản phụ thuộc vào chính trị, văn hóa, xã hội nói chung và nhu cầu của khách hàng nói riêng.

Một số nghiên cứu tìm hiểu mối quan hệ giữa việc áp dụng hệ thống thông tin bất động sản với lợi ích mang lại cho các bên liên quan. Ho và Cook (2013), Lim (2011) đều cho rằng hệ thống thông tin bất động sản có chất lượng tốt ảnh hưởng đáng kể đến lòng tin và sự thỏa mãn của người dùng. Ngoài ra, nghiên cứu của Konieczna (2017) cho thấy việc áp dụng hệ thống thông tin bất động sản mang lại nhiều lợi ích cho quốc gia như quản lý thuế bất động sản tốt hơn, hỗ trợ chiến lược phát triển bất động sản của địa phương...

Tại Việt Nam, có nhiều nghiên cứu về thị trường bất động sản, giá bất động sản và nghề môi giới bất động sản. Tuy nhiên, chưa có nghiên cứu nào về hệ thống thông tin bất động sản đặc biệt là các nhân tố ảnh hưởng đến việc áp dụng hệ thống.

Dựa trên các nghiên cứu của thế giới, tác giả xây dựng mô hình nghiên cứu các nhân tố ảnh hưởng đến việc áp dụng hệ thống thông tin bất động sản (HTTT BĐS) tại tỉnh Tiền Giang. Ngoài ra, tác giả điều chỉnh một số nhân tố cho phù hợp với bối cảnh địa phương.

\section{Cơ sở lý thuyết}

Dựa vào các nghiên cứu trước đây của thế giới, tác giả đã kế thừa và điều chỉnh các nhân tố cho phù hợp với thực tế áp dụng HTTT BĐS tại tỉnh Tiền Giang. Tác giả xác định có ba nhân tố tác động, bao gồm: môi trường kinh doanh, nền tảng kỹ thuật và dịch vụ hỗ trợ. Bên cạnh đó, tác giả cũng kiểm định mối quan hệ giữa việc áp dụng HTTT BĐS và lợi ích áp dụng. Mối quan hệ giả thuyết giữa các biến trong nghiên cứu được mô tả trong Hình 1.

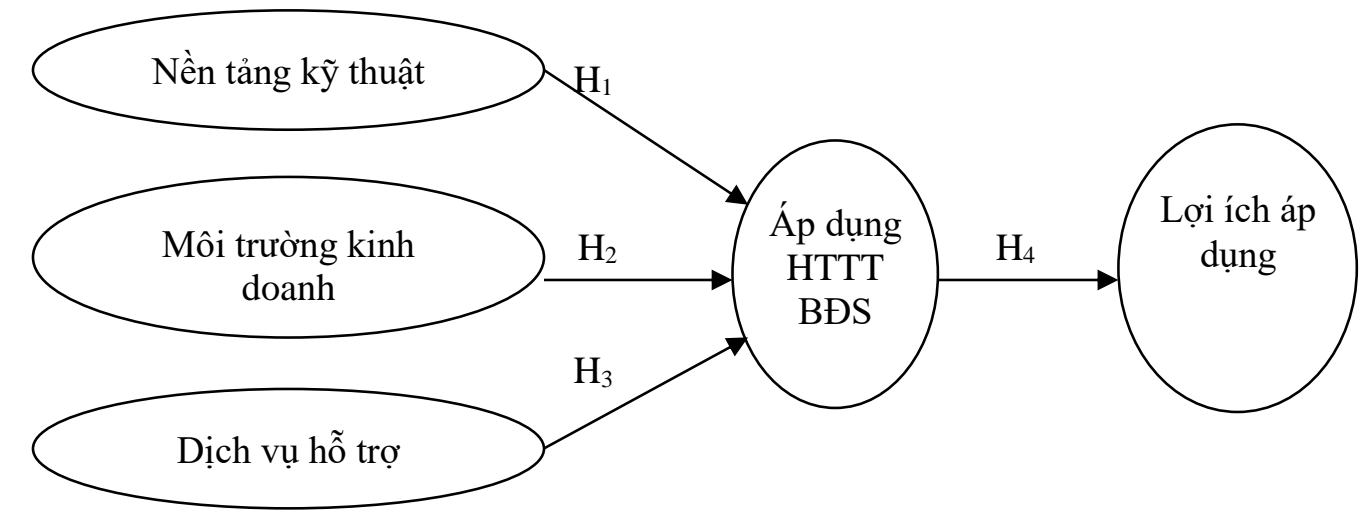

Hình 1. Mô hình nghiên cứu đề xuất

$\mathrm{H}_{1}$ : Nền tảng kỹ thuật có tác động tích cực đến việc áp dụng HTTT BĐS.

$\mathrm{H}_{2}$ : Môi trường kinh doanh có tác động tích cực đến việc áp dụng HTTT BĐS.

$\mathrm{H}_{3}$ : Dịch vụ hỗ trợ có tác động tích cực đến việc áp dụng HTTT BĐS.

$\mathrm{H}_{4}$ : Việc áp dụng HTTT BĐS có tác động tích cực đến lợi ích áp dụng của người dùng. 


\section{Phương pháp nghiên cứu}

Phương pháp hỗn hợp được sử dụng trong nghiên cứu này. Nghiên cứu định tính được thực hiện bằng kỹ thuật thảo luận nhóm và phỏng vấn thử. Sau đó, dựa vào các nghiên cứu trước đây, các biến của thang đo và bảng câu hỏi được xây dựng phù hợp với bối cảnh nghiên cứu tại địa phương. Bảng câu hỏi được phát hành thử và lấy ý kiến phản hồi, sau đó được hiệu chỉnh và làm công cụ thu thập dữ liệu cho nghiên cứu chính thức. Nghiên cứu chính thức được tiến hành theo phương pháp định lượng. Thang đo Likert 5 mức độ (1: Hoàn toàn không đồng ý, 2: Không đồng ý, 3: Trung lập, 4: Đồng ý và 5: Hoàn toàn đồng ý) được sử dụng để đo lường giá trị các biến số.

Đối tượng khảo sát được lựa chọn bằng phương pháp thuận tiện phi xác suất. Thư giới thiệu kèm đường link bảng câu hỏi được gửi tới email cơ quan, công ty hoặc cá nhân của 350 đối tượng khảo sát. Thư nhắc việc được gửi mỗi 2 tuần từ tháng 9/2020 đến tháng 11/2020 để khuyến khích sự phản hồi. Có tất cả 225 đối tượng khảo sát phản hồi thông tin. Tác giả loại bỏ 20 phản hồi không đủ thông tin và 5 phản hồi có giá trị ngoại lai. Vì vậy, còn lại 200 phản hồi hợp lệ được sử dụng để phân tích dữ liệu đạt tỷ lệ $57,14 \%$. Cỡ mẫu này là phù hợp với qui chuẩn nghiên cứu định lượng (Hair, Anderson, Tatham, \& Black, 1998).

Dữ liệu thu thập sẽ được tiến hành phân tích cơ bản và hiệu chỉnh bằng phần mềm SPSS. Mô hình cấu trúc tuyến tính bình phương nhỏ nhất (PLS - SEM) được sử dụng để kiểm định và khẳng định các giả thuyết nghiên cứu. Các bước phân tích được thực hiện dựa trên đề xuất của Frank và Hall (2017), Hair, Hult, Ringle, và Sarstedt (2016), Hwang (2019) và Olesky (2018).

\section{Kết quả nghiên cứu và thảo luận}

\subsection{Mô hình đo lưòng}

Tất cả mô hình đo lường trong nghiên cứu này đều là mô hình đo lường kết quả. Do đó, tác giả đánh giá độ tin cậy của mô hình đo lường dựa trên khuyến nghị của Bagozzi, Yi, và Phillips (1991), Hulland (1999), Hair và cộng sự (2016), Peterson và Kim (2013). Độ chính xác của mô hình đo lường được đánh giá theo khuyến nghị của Hair, Black, Babin, và Anderson (2009), Henseler, Ringle, và Sarstedt (2015).

Bảng 1 trình bày mức độ tin cậy của từng chỉ báo, trong đó, có 2 chỉ báo của thang đo Dịch vụ hỗ trợ bị loại bỏ do hệ số tải ngoài $\lambda<0.4$ (Bagozzi et al., 1991).

\section{Bảng 1}

Mức độ tin cậy từng chỉ báo

\begin{tabular}{|c|c|c|c|c|c|}
\hline & $\begin{array}{c}\text { Áp dụng HTTT } \\
\text { BĐS }\end{array}$ & $\begin{array}{c}\text { Dịch vụ hố } \\
\text { trọ̣ }\end{array}$ & $\begin{array}{c}\text { Lợi ích áp } \\
\text { dụng }\end{array}$ & $\begin{array}{c}\text { Nền tảng kỹ } \\
\text { thuật }\end{array}$ & $\begin{array}{c}\text { Môi trường } \\
\text { kinh doanh }\end{array}$ \\
\hline $\mathrm{AD}_{1}$ 1 & 0.877 & & & & \\
\hline $\mathrm{AD}_{2} 2$ & 0.748 & & & & \\
\hline $\mathrm{AD}$ _3 & 0.835 & & & & \\
\hline $\mathrm{AD}$ _4 & 0.854 & & & & \\
\hline HT_1 & & 0.859 & & & \\
\hline HT_2 & & 0.858 & & & \\
\hline HT_3 & & 0.867 & & & \\
\hline LI_1 & & & 0.826 & & \\
\hline
\end{tabular}




\begin{tabular}{|c|c|c|c|c|c|}
\hline & $\begin{array}{c}\text { Áp dụng HTTT } \\
\text { BĐS }\end{array}$ & $\begin{array}{c}\text { Dịch vụ hố } \\
\text { trọ }\end{array}$ & $\begin{array}{l}\text { Lọi ích áp } \\
\text { dụng }\end{array}$ & $\begin{array}{c}\text { Nền tảng kỹ } \\
\text { thuật }\end{array}$ & $\begin{array}{l}\text { Môi trưò̀ng } \\
\text { kinh doanh }\end{array}$ \\
\hline LI_2 & & & 0.716 & & \\
\hline LI_3 & & & 0.858 & & \\
\hline LI_4 & & & 0.849 & & \\
\hline $\mathrm{KT}_{-} 1$ & & & & 0.759 & \\
\hline $\mathrm{KT} \_2$ & & & & 0.844 & \\
\hline $\mathrm{KT} \_3$ & & & & 0.867 & \\
\hline KD_1 & & & & & 0.867 \\
\hline KD_2 & & & & & 0.840 \\
\hline KD_3 & & & & & 0.811 \\
\hline KD_4 & & & & & 0.767 \\
\hline
\end{tabular}

Nguồn: Kết quả phân tích PLS-SEM (2020)

Kết quả cho thấy các chỉ báo đều có hệ số tải ngoài $\lambda>0.7$, do đó, các chỉ báo đều đạt độ tin cậy (Hair et al., 2016). Tiếp theo, tác giả đánh giá mức độ tin cậy nhất quán nội bộ thông qua chỉ số độ tin cậy tổng hợp (CR). Kết quả chi tiết thể hiện trong Bảng 2.

\section{Bảng 2}

Phân tích độ tin cậy và chính xác thang đo

\begin{tabular}{|l|c|c|c|c|}
\hline & Cronbach's Alpha & rho_A & CR & AVE \\
\hline Áp dụng HTTT BĐS & 0.848 & 0.854 & 0.898 & 0.688 \\
\hline Dịch vụ hỗ trợ & 0.828 & 0.841 & 0.896 & 0.742 \\
\hline Lợi ích áp dụng & 0.832 & 0.854 & 0.887 & 0.663 \\
\hline Nền tảng kỹ thuật & 0.769 & 0.809 & 0.864 & 0.680 \\
\hline Môi trường kinh doanh & 0.842 & 0.863 & 0.893 & 0.676 \\
\hline
\end{tabular}

Nguồn: Kết quả phân tích PLS-SEM (2020)

Theo Bảng 2, các thang đo đều có $0.7<\mathrm{CR}<0.9$, do đó, mô hình đo lường đạt được tính nhất quán nội bộ. Hơn nữa, với Cronbach's Alpha $>0.7$ và $\mathrm{CR}<0.9$ trong tất cả thang đo, có thể kết luận rằng mô hình đo lường thực sự đạt độ tin cậy nhất quán nội bộ (Hair et al., 2016).

Mô hình đo lường của nghiên cứu này đạt độ chính xác về sự hội tụ theo Hair và cộng sự (2009) vì các chỉ báo đều có hệ số tải ngoài $\lambda>0.7$ (Bảng 1 ) và các thang đo đều có mức độ giải thích trung bình của biến tiềm ẩn tới các chỉ báo $\mathrm{AVE}>0.5$ (Bảng 2). Chỉ số tương quan HeterotraitMonostrait (HTMT) được sử dụng để đánh giá độ chính xác về sự phân biệt của mô hình đo lường. Theo kết quả nghiên cứu, hệ số tải ngoài đều lớn hơn hệ số tải chéo của từng chỉ báo. Do đó, điều kiện đánh giá theo chỉ số tương quan HTMT được đảm bảo (Henseler et al., 2015). Các chỉ số tương quan HTMT được trình bày ở Bảng 3 . 


\section{Bảng 3}

Chỉ số tương quan HTMT

\begin{tabular}{|l|c|c|c|c|c|}
\hline & $\begin{array}{c}\text { Áp dụng } \\
\text { HTTT BĐS }\end{array}$ & $\begin{array}{c}\text { Dịch vụ } \\
\text { hỗ trọ́ }\end{array}$ & $\begin{array}{c}\text { Lọi ích áp } \\
\text { dụng }\end{array}$ & $\begin{array}{c}\text { Nền tảng kỹ } \\
\text { thuật }\end{array}$ & $\begin{array}{c}\text { Môi trường } \\
\text { kinh doanh }\end{array}$ \\
\hline Áp dụng HTTT BĐS & & & & & \\
\hline Dịch vụ hỗ trợ & 0.567 & & & & \\
\hline Lợi ích áp dụng & 0.617 & 0.213 & & & \\
\hline Nền tảng kỹ thuật & 0.834 & 0.181 & 0.659 & & \\
\hline Môi trường kinh doanh & 0.677 & 0.594 & 0.262 & 0.398 & \\
\hline
\end{tabular}

Nguồn: Kết quả phân tích PLS-SEM (2020)

Trong từng cặp thang đo trong Bảng 3 đều có chỉ số tương quan $\mathrm{HTMT}<0.85$. Điều này cho thấy các cặp thang đo của mô hình đạt được mức độ về sự chính xác và đủ điều kiện để thực hiện tiến trình boostrapping để thực hiện kiểm định chỉ số HTMT (Henseler et al., 2015). Kết quả thực hiện tiến trình boostrapping 5000 lần cho thấy khoảng tin cậy $95 \%$ của các chỉ số HTMT đều nhỏ hơn 1 . Vì vậy, tập chỉ báo của các thang đo phân biệt với nhau hay mô hình đo lường của nghiên cứu đạt được mức độ chính xác về sự phân biệt (Henseler et al., 2015).

\subsection{Mô hình cấu trúc}

Mô hình cấu trúc được đánh giá mức độ đa cộng tuyến theo ngưỡng đánh giá do Hair, Risher, Sarstedt và Ringle (2019) đề xuất. Độ lớn của hệ số xác định $\left(\mathrm{R}^{2}\right)$ được đánh giá theo khuyến nghị của Henseler và cộng sự (2009). Tầm quan trọng của biến ngoại sinh $\left(\mathrm{f}^{2}\right)$ được đánh giá theo tiêu chuẩn của Cohen (1988). Đánh giá độ chính xác về dự báo của mô hình $\left(\mathrm{Q}^{2}\right)$ theo quy tắc của Hair và cộng sự (2019). Cuối cùng, nghiên cứu đưa ra đánh giá kiểm định các giả thuyết thống kê.

Theo kết quả nghiên cứu, các biến đầu vào của các mô hình đường dẫn đều có hệ số VIF <3. Vì vậy, không xuất hiện hiện tượng đa cộng tuyến trong mô hình cấu trúc (Hair et al., 2019).

Theo kết quả nghiên cứu, biến nội sinh Áp dụng HTTT BĐS trong mô hình có $\mathrm{R}^{2}=0.668>0.5$ (với $\mathrm{P}$ values $=0.000$ ). Do đó, các nhân tố Nền tảng kỹ thuật, Môi trường kinh doanh và Dịch vụ hỗ trợ có mức tác động cao đến Áp dụng HTTT BĐS. Mức tác động của Áp dụng HTTT BĐS đến Lợi ích áp dụng chỉ ở mức trung bình do $0.25<\mathrm{R}^{2}=0.283<0.5$ (với $\mathrm{P}$ values $=0.000$ ). Điều này có thể là do nghiên cứu chỉ tập trung vào một số hữu hạn biến đầu vào (Henseler et al., 2009; Hair et al., 2019).

\section{Bảng 4}

Mức độ giải thích của các biến

\begin{tabular}{|l|c|c|c|}
\hline & $\mathbf{f}^{\mathbf{2}}$ & T Values & P Values \\
\hline Áp dụng HTTT BĐS -> Lợi ích áp dụng & 0.423 & 2.919 & 0.004 \\
\hline Nền tảng kỹ thuật -> Áp dụng HTTT BĐS & 0.903 & 2.989 & 0.003 \\
\hline Dịch vụ hỗ trợ -> Áp dụng HTTT BĐS & 0.162 & 1.966 & 0.049 \\
\hline Môi trường kinh doanh -> Áp dụng HTTT BĐS & 0.150 & 2.024 & 0.043 \\
\hline
\end{tabular}

Nguồn: Kết quả phân tích PLS-SEM (2020) 
Theo Bảng 4, tất cả các biến đầu vào đều có $\mathrm{P}$ values $<0.05$. Vì vậy, các nhân tố Nền tảng kỹ thuật, Môi trường kinh doanh và Dịch vụ hỗ trợ đều có tầm quan trọng trong việc giải thích cho Áp dụng HTTT BĐS và Áp dụng HTTT BĐS đóng vai trò quan trọng trong việc giải thích Lợi ích áp dụng. Đối chiếu f $\mathrm{f}^{2}$ với tiêu chuẩn của Cohen (1988) thì Nền tảng kỹ thuật có mức độ giải thích cao, Môi trường kinh doanh và Dịch vụ hỗ trợ có mức độ giải thích trung bình cho Áp dụng HTTT BĐS. Trong khi đó, Áp dụng HTTT BĐS có mức độ giải thích cao cho Lợi ích áp dụng.

Theo kết quả nghiên cứu, các biến phụ thuộc đều có $\mathrm{Q}^{2}>0$ chứng tỏ năng lực dự báo của mô hình. Hơn nữa, đối chiếu quy tắc của Hair và cộng sự (2019), năng lực dự báo của mô hình chứa biến phụ thuộc Áp dụng HTTT BĐS ở mức trung bình $\left(\mathrm{Q}^{2}=0.449\right)$ và mô hình chứa biến phụ thuộc Lợi ích áp dụng ở mức thấp $\left(\mathrm{Q}^{2}=0.178\right)$.

\section{Bảng 5}

Kết quả kiểm định giả thuyết

\begin{tabular}{|l|c|c|c|c|}
\hline \multicolumn{1}{|c|}{ Giả thuyết } & $\boldsymbol{\beta}$ & T Values & P Values & Suy luận \\
\hline $\mathrm{H}_{1}$ : Nền tảng kỹ thuật -> Áp dụng HTTT BĐS & 0.563 & 9.537 & 0.000 & Chấp nhận \\
\hline $\mathrm{H}_{2}$ : Môi trường kinh doanh -> Áp dụng HTTT BĐS & 0.262 & 4.349 & 0.000 & Chấp nhận \\
\hline $\mathrm{H}_{3}$ : Dịch vụ hỗ trợ -> Áp dụng HTTT BĐS & 0.258 & 3.848 & 0.000 & Chấp nhận \\
\hline $\mathrm{H}_{4}$ : Áp dụng HTTT BĐS -> Lợi ích áp dụng & 0.532 & 8.548 & 0.000 & Chấp nhận \\
\hline
\end{tabular}

Nguồn: Kết quả phân tích PLS-SEM (2020)

Kết quả kiểm định các giả thuyết thông qua tiến trình boostrapping 5.000 mẫu thể hiện ở Bảng 5 . Tất cả mối quan hệ trong các giả thuyết từ $\mathrm{H}_{1}$ đến $\mathrm{H}_{4}$ đều có ý nghĩa thống kê với độ tin cậy $95 \%$.

\subsection{Thảo luận}

Kết quả nghiên cứu cho thấy tất cả giả thuyết từ $\mathrm{H}_{1}$ đến $\mathrm{H}_{4}$ đều được chấp nhận. Do đó, nền tảng kỹ thuật, môi trường kinh doanh và dịch vụ hỗ trợ đều có tác động tích cực đến việc áp dụng HTTT BĐS tại tỉnh Tiền Giang. Trong đó, nền tảng kỹ thuật là có tác động mạnh nhất ( $\beta$ cao, $\mathrm{f}^{2}$ lớn). Môi trường kinh doanh có mức độ giải thích trung bình. Dịch vụ hỗ trợ là nhân tố có tác động yếu nhất. Hơn nữa, áp dụng HTTT BĐS sẽ mang lại lợi ích cho người sử dụng tại tỉnh Tiền Giang.

Kết quả nghiên cứu xác nhận lại rằng nền tảng kỹ thuật có tác động tích cực đến áp dụng HTTT BĐS giống như các nghiên cứu của Fransson và Nelson (2000), Konieczna (2017). Nghiên cứu có sự tương đồng với kết quả của Honda và cộng sự (2018), Kirkwood (2004) khi xác nhận môi trường kinh doanh có tác động tích cực đến việc áp dụng HTTT BĐS. Kết quả nghiên cứu giúp ủng hộ thêm cho nghiên cứu của Fransson và Nelson (2000), Honda và cộng sự (2018) khi xác định dịch vụ hỗ trợ có tác động tích cực đến việc áp dụng HTTT BĐS.

Đây là một trong số các nghiên cứu tiên phong về việc áp dụng HTTT BBĐS tại Việt Nam. Từ đó khẳng định áp dụng HTTT BĐS sẽ mang lại lợi ích cho người sử dụng. Kết quả này góp phần ủng hộ Nghị định 117/2015/NĐ-CP về xây dựng, quản lý và sử dụng hệ thống thông tin về nhà ở và thị trường bất động sản (Chính phủ, 2015). Để áp dụng HTTT BĐS hiệu quả, cần phải chú ý nâng cấp, phát triển cơ sở hạ tầng kỹ thuật và công nghệ. Đặc biệt, bảo mật thông tin là yếu tố cốt lõi quyết định sự thành công và tồn tại bền vững của hệ thống thông tin trong thời đại ngày nay. Bên cạnh đó, các cấp có thẫm quyền cần tạo môi trường kinh doanh thuận lợi và tích hợp 
nhiều tiện ích hỗ trợ bổ sung cho người sử dụng để tăng cường việc áp dụng hệ thống thông tin về nhà ở và thị trường bất động sản.

\section{Kết luận}

Kết quả của nghiên cứu này là hoàn chỉnh các thang đo, kiểm định mô hình, xác định được các nhân tố và mức độ ảnh hưởng đến việc áp dụng HTTT BĐS tại tỉnh Tiền Giang. Qua đó, xác định được ba nhân tố ảnh hưởng là môi trường kinh doanh, nền tảng kỹ thuật và dịch vụ hỗ trợ. Kết quả nghiên cứu cũng khẳng định áp dụng HTTT BĐS sẽ mang lại lợi ích cho người sử dụng.

Nghiên cứu này có một số hạn chế sau đây. Thứ nhất, nghiên cứu chỉ tiến hành trên địa bàn tỉnh Tiền Giang, các nghiên cứu sau có thể mở rộng phạm vi trên toàn quốc. Thứ hai, mô hình cấu trúc của nghiên cứu chỉ xây dựng tác động trực tiếp từ áp dụng HTTT BĐS đến lợi ích áp dụng. Các nghiên cứu tiếp theo có thể xây dựng thêm nhiều nhân tố tác động trực tiếp đến lợi ích áp dụng.

\section{Tài liệu tham khảo}

Bagozzi, R. P., Yi, Y., \& Phillips, L. W. (1991). Assessing construct validity in organizational research. Administrative Science Quarterly, 36(3), 421-458.

Chính Phủ. (2015). Nghị định số 117/2015/NĐ-CP ngày 12 tháng 11 năm 2015 về xây dụng, quản lý và sủ dụng hệ thống thông tin về nhà ở và thị trường bất động sản [Decree No. 117/2015/ ND-CP dated November 12, 2015 on the construction, management and use of information systems on housing and real estate market]. Retrieved May 10, 2019, from https://thuvienphapluat.vn/van-ban/Bat-dong-san/Nghi-dinh-117-2015-ND-CP-xay-dungquan-ly-su-dung-he-thong-thong-tin-ve-nha-o-thi-truong-bat-dong-san-297203.aspx

Cohen, J. (1988). Statistical power analysis for the behavioral sciences (2nd ed.). Mahwah, US: Lawrence Erlbaum Associates.

Frank, E., \& Hall, M. A. (2017). Data mining: Practical machine learning tools and techniques (4th ed.). Burlington, MA: Morgan Kaufmann.

Fransson, W., \& Nelson, D. (2000). Management information systems for corporate real estate. Journal of Corporate Real Estate, 2(2), 154-169.

Hair, J. F. J., Anderson, R. E., Tatham, R. L., \& Black, W. C. (1998). Multivariate data analysis (5th ed.). Hoboken, NJ: Prentice Hall College Div.

Hair, J. F., Black, W., Babin, B., \& Anderson, R. (2009). Multivariate data analysis (7th ed.). Hoboken, NJ: Pearson Prentice Hall.

Hair, J. F., Hult, G. T. M., Ringle, C. M., \& Sarstedt, M. (2016). A primer on Partial Least Squares Structural Equation Modeling (PLS-SEM) (1st ed.). Thousand Oaks, CA: Sage publications.

Hair, J. F., Risher, J. J., Sarstedt, M., \& Ringle, C. M. (2019). When to use and how to report the results of PLS-SEM. European Business Review, 31(1), 2-24.

Henseler, J., Ringle, C. M., \& Sarstedt, M. (2015). A new criterion for assessing discriminant validity in variance-based structural equation modeling. Journal of the Academy of Marketing Science, 43(1), 115-135.

Henseler, J., Ringle, C. M., \& Sinkovics, R. R. (2009). The use of partial least squares path modeling in international marketing. Advances in International Marketing, 20, 277-319. 
Ho, L. C., \& Cook, M. H. (2013). The effect of real estate information systems quality on system use and user satisfaction. The Journal of Digital Policy \& Management, 11(11), 243-249.

Honda, H., Sasaki, S., Hayashi, Y., \& Yasushi, K. (2018). A regional-diversity-corresponding real estate information search \& evaluation system. In 2018 International Electronics Symposium on Knowledge Creation and Intelligent Computing (IES-KCIC). Surabaya, East Java, Indonesia.

Hulland, J. (1999). Use of Partial Least Squares (PLS) in strategic management research: A review of four recent studies. Strategic Management Jounal, 20(2), 195-204.

Hwang, Y. H. (2019). Hands-on data science for marketing: Improve your marketing strategies with machine learning using Python and R. Birmingham, UK: Packt Publishing.

Kirkwood, J. S. (2004). Urban real estate information systems: The suppression of radical innovation. Journal of Urban Technology, 11(1), 29-42.

Konieczna, J. (2017). The cadastre with respect to the integrated system of real estate information. In Environmental Engineering 10th International Conference. Vilnius, Lithuania: Vilnius Gediminas Technical University.

Lim, C. K. (2011). A relationship among service quality, trust, customer satisfaction and reuse intention in real estate information web-sites. Journal of the Korea Industrial Information Systems Research, 16(2), 125-137.

Olesky, A. (2018). Data Science with R: A step by step guide with visual illustrations \& examples. Traverse City, MI: Independently published.

Peterson, R. A., \& Kim, Y. (2013). On the relationship between coefficient alpha and composite reliability. Journal of Applied Psychology, 98(1), 194-198. 